\title{
Röntgenologische Untersuchungsmethode von Pflanzen und Pflanzenteilen für züchterische $Z$ wecke.
}

\author{
(Vorläufige Mitteilung.) \\ Von R. Freisleben-Halle, E. A. W. Müller-Berlin, R. v. Sengbusch-Luckenwalde.
}

Der Züchter wird bei den verschiedensten Objekten vor die Aufgabe gestellt, eine Auslese nach morphologischen Gesichtspunkten vorzunehmen. Wenn sich die morphologischen Merkmale äußerlich ausprägen, bereitet die Auslese keine Schwierigkeit. Wenn der Züchter aber den morphologischen Aufbau innerhalb einer Pflanze zum Gegenstand seiner Auslese macht, so kann er diesen Aufbau erst durch Aufschneiden oder Zerlegen der Pflanze sichtbar machen.

v. SengBusch hat infolgedessen die Frage aufgeworfen, ob es durch eine röntgenologische Untersuchung möglich sei, den inneren morphologischen Aufbau von Pflanzen und Pflanzenteilen sichtbar zu machen. In der Röntgenabteilung der Firma Siemens \& Halske, die unter der Leitung von E. A. W. MÜLlER steht, wurden im Oktober I 942 die ersten diesbezüglichen Versuche durchgeführt.

Die röntgenologische Aufnahme eines Kohlkopfes zeigte, daß man den Aufbau, der sonst nur an Querschnitten von Kohl erkennbar wird, auf dem Röntgenbild deutlich wahrnehmen kann:

I. die aufeinander gelagerten Blätter in dem oberen Drittel des Kohls,

2. die Strunkform,

3. die Ansätze der Blattstiele am Strunk.

Auch an anderen Objekten, wie z. B. am Apfel, wurden bei einer Durchleuchtung auf dem Schirm der Aufbau des Apfelgehäuses sowie die Lage der Kerne sichtbar gemacht.

Bei der Durchleuchtung von Holz konnte ebenfalls die Struktur auf dem Leuchtschirm erkannt werden.
Diese ersten Versuche deuten darauf hin, daß es möglich sein wird, durch Röntgenaufnahmen und Röntgenschirmbilder den inneren Aufbau der verschiedensten Objekte sichtbar zu machen, so daß der Pflanzenzüchter in der Lage sein wird, auf Grund dieser Bilder eine Auslese vorzunehmen. Im nachfolgenden führen wir eine $\mathrm{Zu}$ sammenstellung derjenigen Objekte auf, die für die röntgenologische Untersuchung in Frage kommen: I. Der Aubau des Kopfkohls. 2. Der Aufbau der Möhre; Sichtbarmachung von Rindenteil, Kambiumzone und Holzteil sowie der Wurzelansätze im Rindenteil. 3. Tomaten; Zahl der Kammern und Samen. 4. Der Aufbau der Zwiebel. 5. Der Aufbau von Kopfsalat. 6. Hanf und Lein; Sichtbarmachung der Faserzellen. 7. Getreide; Sichtbarmachung des Stützgewebes. 8. Bespelzung des Kornes. 9. Citrusfrüchte und Reben; Sichtbarmachung der Samenmenge und bei Citrusfrüchten des Aufbaues der Früchte. Io. Forstpflanzen; Struktur des Holzes. Diese Aufstellung kann naturgemäß nicht vollständig sein. Wir wollen damit nur andeuten, welche Anwendungsmöglichkeiten der röntgenologischen Untersuchung für züchterische Zwecke offen stehen.

Auf die Technik der Durchführung derartiger röntgenologischer Untersuchungen für züchterische Zwecke wird in einer späteren Veröffentlichung näher eingegangen werden.

Wir hoffen, da $B$ dieses neue Gebiet der Pflanzenuntersuchung dem Züchter ein neues Hilfsmittel für erfolgreiche Arbeit bieten wird. 